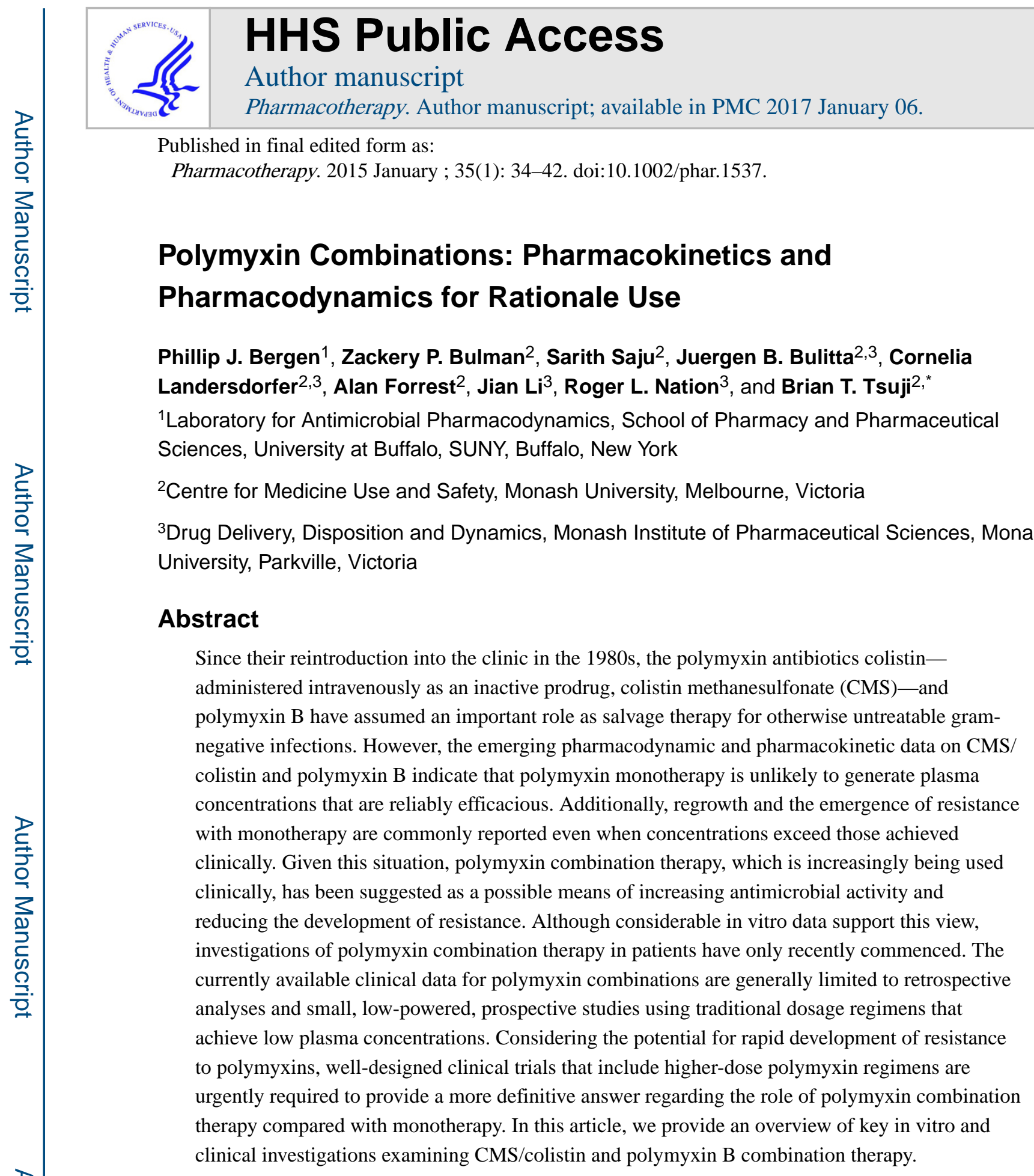

\title{
Keywords
}

colistin; colistin methanesulfonate; polymyxins; polymyxin B; combination

The polymyxin antibiotics colistin-administered intravenously as colistin methanesulfonate (CMS), the sulfomethylated derivative and prodrug of colistin — and polymyxin B were first

*Address for correspondence: Brian T. Tsuji, NYS Center of Excellence in Bioinformatics and Life Sciences, 701 Ellicott Street, Buffalo, NY 14203; btsuji@buffalo.edu. 
used clinically in the 1950s before toxicity concerns, primarily nephrotoxicity and neurotoxicity, led to a substantial decline in their use. ${ }^{1,2}$ However, with few novel antimicrobial agents in development ${ }^{3}$ and an increasing incidence of infections caused by multidrug-resistant (MDR) gram-negative organisms, ${ }^{4}$ polymyxins are increasingly being used as salvage therapy for otherwise untreatable infections. Recent pharmacodynamic (PD) and pharmacokinetic (PK) data on CMS/colistin and polymyxin B suggest that polymyxin monotherapy is unlikely to generate reliably efficacious plasma concentrations ${ }^{5,6}$; additionally, in vitro regrowth and the emergence of resistance with monotherapy may occur even when concentrations greatly exceed those achieved clinically. ${ }^{7,8}$ Consequently, polymyxin combination therapy has been suggested as a way to increase antimicrobial activity and reduce the emergence of resistance, ${ }^{5,9}$ and, indeed, it is increasingly being used clinically. ${ }^{10-17}$ However, combination therapy has potential disadvantages including increased cost and risk of drug toxicity. ${ }^{18}$ In this article, we provide an overview of key in vitro and clinical investigations examining CMS/colistin and polymyxin B combination therapy; other aspects of polymyxin pharmacology are reviewed elsewhere. ${ }^{19}$ As significant shortcomings exist with current animal studies, these are not reviewed.

\section{In vitro Studies}

Time-kill methods, both static and PK/PD (dynamic), use serial viable counting to provide a picture of antimicrobial activity over time and thus have an important advantage over the more commonly used fractional inhibitory concentration (FIC) index and Etest methods, which provide only inhibitory data at a single time point; consequently, only time-kill studies are reviewed in this article. In time-kill studies, synergy and antagonism are most commonly defined as a 100-fold increase or decrease, respectively, in killing at 24 hours (as measured by colony counts) with the combination relative to its most active component, although variations of these definitions (e.g., at times other than $24 \mathrm{hrs}$ ) abound. For the polymyxins, most existing combination studies use colistin rather than polymyxin B, most likely because of colistin's wider geographical use. Pseudomonas aeruginosa, Acinetobacter baumannii, and Klebsiella pneumoniae are the organisms most commonly studied and are the focus of this review.

\section{Static Time-Kill Studies}

Pseudomonas aeruginosa-A study investigated bacterial killing and resistance emergence over 48 hours with nine colistin/imipenem combinations (the majority using clinically achievable concentrations) against five clinical isolates and a reference strain at two inocula $\left(\sim 10^{6}\right.$ and $\sim 10^{8}$ colony-forming units $\left.[\mathrm{CFU}] / \mathrm{ml}\right)$; isolates included colistin- and imipenem-susceptible and -resistant strains, and MDR and non-MDR strains. ${ }^{20}$ Regrowth occurred with colistin monotherapy $(0.5 \times$ minimum inhibitory concentration [MIC], $4 \times$ MIC, and 16× MIC for susceptible isolates; 1,4 and $32 \mathrm{mg} / \mathrm{L}$ for resistant isolates) with all isolates (Figure 1). However, the addition of imipenem ( $0.5 \times \mathrm{MIC}, 4 \times \mathrm{MIC}$, and $16 \times \mathrm{MIC}$ for susceptible isolates; 1,8 and $32 \mathrm{mg} / \mathrm{L}$ for resistant isolates) to colistin at both inocula generally resulted in substantial improvements in bacterial killing over equivalent monotherapy across 48 hours against MDR isolates resistant to either antibiotic (Figure 1); enhanced killing also occurred across approximately the first 6 hours against isolates 
susceptible to both antibiotics but was less pronounced at later time points. At each inoculum, colistin resistance in colistin-susceptible isolates emerged with both colistin monotherapy and combination therapy in a similar fashion (Figure 1B,C, right panels); however, this result is in contrast to later studies using PK/PD models (discussed below). Other studies combined colistin $(0.12-16 \mathrm{mg} / \mathrm{L})$ with meropenem $(0.06-8 \mathrm{mg} / \mathrm{L})^{21}$ or doripenem $(0.03-128 \mathrm{mg} / \mathrm{L}){ }^{22}$ Synergy with sub-MIC concentrations was reported against $13(25.5 \%)$ of 51 isolates of $P$. aeruginosa at 24 hours with colistin/meropenem combinations (colistin MIC 0.25-2 mg/L, meropenem MIC 0.12-256 mg/L) and 19 (76.0\%) of 25 isolates with colistin/doripenem combinations (colistin MIC 0.12-32 mg/L, doripenem MIC $0.12-256 \mathrm{mg} / \mathrm{L}$ ); a majority of isolates in the latter study were included in the former study.

Acinetobacter baumannii-These studies also combined colistin with meropenem ${ }^{21}$ or doripenem $^{22}$ against clinical isolates of $A$. baumannii. Colistin $(0.06-8 \mathrm{mg} / \mathrm{L})$ and meropenem $(0.03-64 \mathrm{mg} / \mathrm{L})$ showed synergy against 49 (94.2\%) of 52 isolates (colistin MIC $0.12-128 \mathrm{mg} / \mathrm{L}$, meropenem MIC $0.12-256 \mathrm{mg} / \mathrm{L})$ at 24 hours, whereas colistin $(0.12-16$ $\mathrm{mg} / \mathrm{L})$ and doripenem $(0.06-32 \mathrm{mg} / \mathrm{L})$ showed synergy against 25 (100\%) of 25 isolates (colistin MIC $0.12-32 \mathrm{mg} / \mathrm{L}$, doripenem MIC $0.25-128 \mathrm{mg} / \mathrm{L}$ ); a majority of isolates in the latter study were included in the former study. Another study ${ }^{23}$ examined colistin plus doripenem against five extensively drug-resistant (XDR; defined as resistant to all agents except polymyxins and tigecycline) but colistin-susceptible isolates of $A$. baumannii taken from solid organ transplant recipients. Colistin monotherapy $(0.25-1 \times \mathrm{MIC})$ was bacteriostatic against all isolates, but the combination of colistin $(0.125-0.259 \times \mathrm{MIC})$ plus doripenem $(8 \mathrm{mg} / \mathrm{L})$ resulted in undetectable bacterial concentrations at 8 hours without evidence of regrowth by 24 hours (Figure 2 ).

One study examined colistin $(6 \mathrm{mg} / \mathrm{L})$, rifampin $(5 \mathrm{mg} / \mathrm{L})$, imipenem $(20 \mathrm{mg} / \mathrm{L})$ and ampicillin/sulbactam $(50 \mathrm{mg} / \mathrm{L})$ alone or in double (colistin plus each of the second drugs) or triple (colistin/rifampin/imipenem, or colistin/rifampin/ampicillin/sulbactam) combinations against nine isolates of MDR A. baumannii-producing oxacillinase (OXA)-58 carbapenemase. ${ }^{24}$ Colistin was most active with double and triple combinations generally having similar activity to colistin monotherapy. However, in another study, triple therapy with polymyxin $\mathrm{B}$, doripenem, and rifampin was more effective than monotherapy or double combination therapy against five non-metallo- $\beta$-lactamase (MBL)- or Klebsiella pneumoniae carbapenemase (KPC)-producing isolates; all isolates were polymyxin B susceptible and doripenem resistant. ${ }^{25}$ Against five MDR colistin-susceptible isolates of $A$. baumannii, colistin $(1 \mathrm{mg} / \mathrm{L})$ plus vancomycin $(20 \mathrm{mg} / \mathrm{L})$ or teicoplanin $(20 \mathrm{mg} / \mathrm{L})$ dramatically suppressed regrowth in 4 of 5 isolates (vancomycin) or all isolates (teicoplanin) compared to monotherapy, with $\sim 5$-fold to more than 8 -fold $\log _{10} \mathrm{CFU} / \mathrm{ml}$ greater killing at 24 hours. ${ }^{26,27}$ It has been suggested that the observed activity against gram-negative bacteria in such circumstances by antibiotics normally inactive against these organisms (e.g., glycopeptides, rifampin, and macrolides) may be due to substantial changes in the outer membrane occurring as a result of colistin resistance, increasing membrane permeability. ${ }^{28}$ 
Klebsiella pneumoniae and Other Enterobacteriaceae-A study examined colistin plus tigecycline against eight KPC-producing enterobacterial clinical strains (four $K$. pneumoniae, two Escherichia coli, one Enterobacter cloacae, and one Serratia marcescens); all produced KPC-2 carbapenemase and were susceptible to colistin (MIC 0.5-1 mg/L) and (with one exception) tigecycline (MIC $0.25-4 \mathrm{mg} / \mathrm{L}$ ). ${ }^{29}$ Each antibiotic was tested at $1 \times$ MIC, $2 \times$ MIC, and $4 \times$ MIC. Combinations substantially improved bacterial killing across 24 hours and were synergistic at $1 \times$ MIC and $2 \times$ MIC against most organisms at 4 and 8 hours; at $4 \times$ MIC, synergy was reported at 24 hours against all strains. Another study ${ }^{30}$ examined colistin ( $2 \mathrm{mg} / \mathrm{L}$ ) plus doripenem ( $8 \mathrm{mg} / \mathrm{L}$ ) against $23 \mathrm{KPC}-2$-producing strains of $K$.

pneumoniae, each containing a variant mutant $o p m K 35$ porin gene. The median colistin and doripenem MICs were $4 \mathrm{mg} / \mathrm{L}$ (range 0.125-128 mg/L) and $32 \mathrm{mg} / \mathrm{L}$ (range 4-256 mg/L), respectively. Colistin MICs were $>2 \mathrm{mg} / \mathrm{L}$ against 14 of 23 strains. The combination was synergistic at 24 hours against four strains with doripenem MICs of $\$ 8 \mathrm{mg} / \mathrm{L}$, although there was no overall difference in median bacterial killing for strains with doripenem MICs $>8$ $\mathrm{mg} / \mathrm{L}$ (synergy in 6 [32\%] of 19 strains). Notably, insertions encoding glycine and aspartic acid at amino acid (aa) positions 134 and 135 (ins aa134-135 GD [n=8]) and ompK36 promoter IS 5mutations $(\mathrm{n}=7)$ were associated with significantly higher doripenem MICs and diminished efficacy of colistin/doripenem combinations.

Fewer studies address MBL-producing strains compared to KPC-producing strains. One examined colistin $(5 \mathrm{mg} / \mathrm{L})$ plus imipenem $(10 \mathrm{mg} / \mathrm{L})$ against 42 unique clinical isolates of bla Verona integrin-encoded metallo- $\beta$-lactamase (VIM)-1-type MBL-producing $K$. pneumoniae. ${ }^{31}$ Synergy at 24 hours was reported against 12 (50\%) of 24 colistin-susceptible isolates, but antagonism was observed against $10(55.6 \%)$ of 18 colistin-resistant isolates. Another study ${ }^{32}$ conducted over 200 time-kill experiments against two VIM-1-type and two New Delhi metallo- $\beta$-lactamase (NDM)-1-type $K$. pneumoniae strains (all colistin susceptible, MIC $0.125 \mathrm{mg} / \mathrm{L}$ ). At 24 hours, colistin plus fosfomycin (MIC 4-256 mg/L) was bactericidal and synergistic against three of the four strains (both NDM-1-types [each fosfomycin resistant] and one VIM-1-type), whereas the triple combination of colistin/ fosfomycin/meropenem was bactericidal against three and synergistic against all strains.

\section{Pharmacokinetic and Pharmacodynamic Studies}

Three studies used one-compartment PK/PD models to mimic the PK of antibiotics in patients. These examined killing of planktonic (free-floating) bacteria at two inocula ( $10^{6}$ and $\sim 10^{8} \mathrm{CFU} / \mathrm{ml}$ ) across $72-96$ hours. ${ }^{33-35}$ All employed clinically relevant concentrations of colistin administered as a continuous infusion to simulate the "flat" profiles of formed colistin observed in critically ill patients at steady state after CMS administration. 5, 36 Colistin (constant concentrations of 0.5 or $2 \mathrm{mg} / \mathrm{L}$ ) was combined with doripenem (maximum concentration $\left[\mathrm{C}_{\max }\right]$ of 2.5 or $25 \mathrm{mg} / \mathrm{L}$ given every $8 \mathrm{~h}$; half-life $1.5 \mathrm{hrs}$ ) against MDR $P$. aeruginos $a^{33}$ and $K$. pneumoniae, ${ }^{35}$ and with an additional colistin concentration of $5 \mathrm{mg} / \mathrm{L}$, with rifampin $\left(\mathrm{C}_{\max }\right.$ of $5 \mathrm{mg} / \mathrm{L}$ given every $24 \mathrm{~h}$; half-life $\left.3 \mathrm{hrs}\right)$ against MDR $A$. baumannii. ${ }^{34}$ In all cases, improvements in bacterial killing were generally observed across 72-96 hours at both inocula (Figure 3, left panels). Improvements were often dramatic, with no viable bacteria detected on at least one occasion at both inocula against all three bacterial species; some combinations even resulted in eradication of a MDR-colistin-resistant isolate 
of $P$. aeruginosa at the low inoculum. ${ }^{33}$ Importantly and in contrast to the static time-till investigation discussed previously ${ }^{20}$ (Figure 1), colistin combination therapy in all cases substantially suppressed or eliminated the emergence of colistin resistance (Figure 4). Recently, enhanced bacterial killing and suppression of the emergence of colistin resistance has also been reported with colistin (constant concentrations of 1.25 or $3.50 \mathrm{mg} / \mathrm{L}$ ) combined with doripenem $\left(\mathrm{C}_{\max }\right.$ of $25 \mathrm{mg} / \mathrm{L}$ given every $8 \mathrm{~h}$; half-life $\left.1.5 \mathrm{hrs}\right)$ against biofilm-embedded MDR P. aeruginosa. ${ }^{37}$

\section{Clinical Studies of CMS or Polymyxin B Combination Therapy}

Clinical data for CMS or polymyxin B are generally limited to retrospective analyses and small, low-power, prospective studies, with studies examining the efficacy of polymyxin combination therapy versus monotherapy virtually absent from the literature. Consequently, most data reviewed below are from investigations that examined the general clinical benefits of polymyxin therapy and provided the greatest insights into specific situations where polymyxin combination therapy appears to be of promise or significant value.

For $K$. pneumoniae, studies have demonstrated reduced mortality with combination therapy compared to monotherapy for KPC-producing $K$. pneumoniae bacteremia, especially with colistin or polymyxin B combined with a carbapenem or tigecycline. ${ }^{38-40}$ In a retrospective cohort analysis examining KPC-producing $K$. pneumoniae bacteremia, 19 patients received monotherapy, with most receiving CMS or polymyxin B (7 patients), tigecycline (5 patients), or a carbapenem (imipenem or meropenem [4 patients]), whereas 15 patients received antibiotic combinations (CMS or polymyxin B combined with unspecified carbapenems [5 patients], tigecycline [1 patient] or a fluoroquinolone [1 patient]; polymyxin-free combinations included tigecycline plus a carbapenem [3 patients] or an aminoglycoside [2 patients]). ${ }^{38}$ Combination treatment was the only significant predictor of survival, with a 28 -day mortality of $13.3 \%$ (2/15 patients) for monotherapy compared to $57.8 \%$ (11/19 patients) for combinations. Notably, 1 (14.2\%) of 7 patients receiving polymyxin combination therapy died compared with 4 (57.1\%) of 7 patients receiving polymyxin monotherapy. One study ${ }^{40}$ similarly reported a significant reduction in 30-day mortality with combination therapy compared to monotherapy for KPC-producing $K$. pneumoniae bacteremia. In this study, 51 patients received CMS combination therapy (mainly combined with tigecycline, gentamicin, or meropenem), and 22 patients received CMS monotherapy. Unfortunately, individual mortality rates for each combination regimen were not stated. Although polymyxin combination therapy may be of benefit for severe infections such as bacteremia, the cumulative assessment of all infection types, including patients considerably less ill, suggests that polymyxin monotherapy may be sufficient. ${ }^{41}$

Few studies examined polymyxin combinations against $P$. aeruginosa. One study prospectively compared CMS monotherapy (10 patients) and combination therapy (13 patients) with amikacin or an antipseudomonal $\beta$-lactam in patients infected with MDR $P$. aeruginosa; infections were diverse and included pneumonia, bacteremia, and intraabdominal infections. ${ }^{42}$ No significant difference in response was noted between the groups, although coinfection with other pathogens in 11 patients may have confounded the results. A similar study involving MDR $P$. aeruginosa likewise concluded that polymyxin B 
combinations (mainly with imipenem) did not provide additional benefit over polymyxin B monotherapy for pneumonia. ${ }^{43}$ Overall, the limited existing clinical data do not support the use of polymyxin combination therapy over monotherapy for treatment of infections with MDR $P$. aeruginosa.

For A. baumannii, two recent prospective studies that compared CMS monotherapy to a CMS/rifampin combination for treatment of ventilator-associated pneumonia (VAP) ${ }^{16}$ or diverse infections (mainly VAP but also bloodstream infections, hospital-acquired pneumonia, and intraabdominal infections) ${ }^{14}$ caused by $A$. baumannii found no significant difference in clinical response or 30-day mortality, respectively, between the two groups. However, microbiological clearance was obtained significantly more quickly with combination therapy (mean \pm SD $4.5 \pm 1.7$ days for monotherapy, $3.1 \pm 0.5$ days for combination) in the first study, ${ }^{16}$ whereas eradication of $A$. baumannii was significantly higher with the addition of rifampin (60.6\% vs $44.8 \%)$ in the second study. ${ }^{14}$ At present, existing clinical data do not show a benefit with polymyxin combination therapy for treatment of MDR A. baumannii.

Although the potential benefits of polymyxin combination therapy over monotherapy suggested by in vitro data are less apparent in existing clinical studies (as discussed above), it is important to recognize that virtually all clinical studies using CMS (the majority of clinical studies) administer CMS in a traditional manner; such administration is unlikely to achieve colistin plasma concentrations in many patients sufficient to treat severe infections. ${ }^{5,6}$ Recent studies suggest the use of a CMS loading dose followed by 9 million units/day in divided doses. ${ }^{44-47}$ The low concentrations obtained with currently employed dosage regimens may explain why studies examining polymyxin use for a variety of MDR gram-negative pathogens and infection sites have been indecisive in differentiating between the value of monotherapy and combination therapy. ${ }^{46,48-51}$ Given the limitations associated with existing clinical data, future randomized controlled trials with robust study designs and that will include higher-dose polymyxin regimens are urgently required to more fully understand the utility of CMS- or polymyxin B-based combinations.

\section{Conclusion}

Although polymyxins retain activity against many problematic MDR gram-negative organisms, the emergence of polymyxin resistance during monotherapy is worrisome. Attention has now shifted toward identifying antibiotic combinations that increase bacterial killing and suppress the emergence of resistance. Whereas in vitro data suggest potential clinical benefits with many polymyxin combinations at clinically achievable concentrations, the practical and ethical considerations involved when undertaking clinical investigations comparing polymyxin monotherapy and combination therapy means that the existing clinical data on polymyxin combination therapy are inconclusive. Well-designed clinical trials are required to elucidate the true value of polymyxin combinations in patients. Given the ethical and logistical challenges of conducting such investigations, however, data derived from preclinical models will continue to provide essential information toward optimization of polymyxin therapy. 


\section{Acknowledgments}

This work was supported in part by the National Institutes of Health, National Institute of Allergy and Infectious Diseases (R01AI079330 and R01AI111990).

\section{References}

1. Koch-Weser J, Sidel VW, Federman EB, Kanarek P, Finer DC, Eaton AE. Adverse effects of sodium colistimethate. Manifestations and specific reaction rates during 317 courses of therapy. Ann Intern Med. 1970; 72:857-68. [PubMed: 5448745]

2. Yow EM, Moyer JH. Toxicity of polymyxin B. II. Human studies with particular reference to evaluation of renal function. AMA Arch Intern Med. 1953; 92:248-57. [PubMed: 13079346]

3. Walker B, Barrett S, Polasky S, et al. Environment. Looming global-scale failures and missing institutions. Science. 2009; 325:1345-6. [PubMed: 19745137]

4. World Health Organization. Antimicrobial resistance: global report on surveillance 2014. Geneva, Switzerland: World Health Organization; 2014.

5. Garonzik SM, Li J, Thamlikitkul V, et al. Population pharmacokinetics of colistin methanesulfonate and formed colistin in critically ill patients from a multicenter study provide dosing suggestions for various categories of patients. Antimicrob Agents Chemother. 2011; 55:3284-94. [PubMed: 21555763]

6. Sandri AM, Landersdorfer CB, Jacob J, et al. Population pharmacokinetics of intravenous polymyxin B in critically ill patients: implications for selection of dosage regimens. Clin Infect Dis. 2013; 57:524-31. [PubMed: 23697744]

7. Bergen PJ, Li J, Nation RL, Turnidge JD, Coulthard K, Milne RW. Comparison of once-, twice- and thrice-daily dosing of colistin on antibacterial effect and emergence of resistance: studies with Pseudomonas aeruginosa in an in vitro pharmacodynamic model. J Antimicrob Chemother. 2008; 61:636-42. [PubMed: 18227094]

8. Gunderson BW, Ibrahim KH, Hovde LB, Fromm TL, Reed MD, Rotschafer JC. Synergistic activity of colistin and ceftazidime against multiantibiotic-resistant Pseudomonas aeruginosa in an in vitro pharmacodynamic model. Antimicrob Agents Chemother. 2003; 47:905-9. [PubMed: 12604520]

9. Rahal JJ. Novel antibiotic combinations against infections with almost completely resistant Pseudomonas aeruginosa and Acinetobacter species. Clin Infect Dis. 2006; 43(Suppl 2):S95-9. [PubMed: 16894522]

10. Shields RK, Clancy CJ, Gillis LM, et al. Epidemiology, clinical characteristics and outcomes of extensively drug-resistant Acinetobacter baumannii infections among solid organ transplant recipients. PLoS ONE. 2012; 7:e52349. [PubMed: 23285002]

11. Garnacho-Montero J, Amaya-Villar R, Gutierrez-Pizarraya A, et al. Clinical efficacy and safety of the combination of colistin plus vancomycin for the treatment of severe infections caused by carbapenem-resistant Acinetobacter baumannii. Chemotherapy. 2013; 59:225-31. [PubMed: 24356297]

12. Petrosillo N, Giannella M, Antonelli M, et al. Clinical experience of colistin-glycopeptide combination in critically ill patients infected with gram-negative bacteria. Antimicrob Agents Chemother. 2014; 58:851-8. [PubMed: 24277037]

13. Morelli P, Ferrario A, Tordato F, Piazza A, Casari E. Successful treatment of post-neurosurgical multidrug-resistant Pseudomonas aeruginosa meningo-encephalitis with combination therapy of colistin, rifampicin and doripenem. J Antimicrob Chemother. 2014; 69:857-9. [PubMed: 24169582]

14. Durante-Mangoni E, Signoriello G, Andini R, et al. Colistin and rifampicin compared with colistin alone for the treatment of serious infections due to extensively drug-resistant Acinetobacter baumannii: a multicenter, randomized clinical trial. Clin Infect Dis. 2013; 57:349-58. [PubMed: 23616495]

15. Ceccarelli G, Falcone M, Giordano A, et al. Successful ertapenem-doripenem combination treatment of bacteremic ventilator-associated pneumonia due to colistin-resistant KPC-producing Klebsiella pneumoniae. Antimicrob Agents Chemother. 2013; 57:2900-1. [PubMed: 23571536] 
16. Aydemir H, Akduman D, Piskin N, et al. Colistin vs. the combination of colistin and rifampicin for the treatment of carbapenem-resistant Acinetobacter baumannii ventilator-associated pneumonia. Epidemiol Infect. 2013; 141:1214-22. [PubMed: 22954403]

17. Batirel A, Balkan II, Karabay O, et al. Comparison of colistin-carbapenem, colistin-sulbactam, and colistin plus other antibacterial agents for the treatment of extremely drug-resistant Acinetobacter baumannii bloodstream infections. Eur J Clin Microbiol Infect Dis. 2014; 33:1311-22. [PubMed: 24532009]

18. Moore NM, Flaws ML. Treatment strategies and recommendations for Pseudomonas aeruginosa infections. Clin Lab Sci. 2011; 24:52-6. [PubMed: 21404966]

19. Bergen PJ, Landersdorfer CB, Zhang J, et al. Pharmacokinetics and pharmacodynamics of "old" polymyxins: what is new? Diagn Microbiol Infect Dis. 2012; 74:213-23. [PubMed: 22959816]

20. Bergen PJ, Forrest A, Bulitta JB, et al. Clinically relevant plasma concentrations of colistin in combination with imipenem enhance pharmacodynamic activity against multidrug-resistant Pseudomonas aeruginosa at multiple inocula. Antimicrob Agents Chemother. 2011; 55:5134-42. [PubMed: 21876058]

21. Pankuch GA, Lin G, Seifert H, Appelbaum PC. Activity of meropenem with and without ciprofloxacin and colistin against Pseudomonas aeruginosa and Acinetobacter baumannii. Antimicrob Agents Chemother. 2008; 52:333-6. [PubMed: 17967915]

22. Pankuch GA, Seifert H, Appelbaum PC. Activity of doripenem with and without levofloxacin, amikacin, and colistin against Pseudomonas aeruginosa and Acinetobacter baumannii. Diagn Microbiol Infect Dis. 2010; 67:191-7. [PubMed: 20338710]

23. Shields RK, Kwak EJ, Potoski BA, et al. High mortality rates among solid organ transplant recipients infected with extensively drug-resistant Acinetobacter baumannii: using in vitro antibiotic combination testing to identify the combination of a carbapenem and colistin as an effective treatment regimen. Diagn Microbiol Infect Dis. 2011; 70:246-52. [PubMed: 21353436]

24. Tripodi MF, Durante-Mangoni E, Fortunato R, Utili R, Zarrilli R. Comparative activities of colistin, rifampicin, imipenem and sulbactam/ampicillin alone or in combination against epidemic multidrug-resistant Acinetobacter baumannii isolates producing OXA-58 carbapenemases. Int J Antimicrob Agents. 2007; 30:537-40. [PubMed: 17851050]

25. Urban C, Mariano N, Rahal JJ. In Vitro double and triple bactericidal activities of doripenem, polymyxin B, and rifampin against Multidrug-resistant Acinetobacter baumannii, Pseudomonas aeruginosa, Klebsiella pneumoniae, and Escherichia coli. Antimicrob Agents Chemother. 2010; 54:2732-4. [PubMed: 20368401]

26. Gordon NC, Png K, Wareham DW. Potent synergy and sustained bactericidal activity of a vancomycin-colistin combination versus multidrug-resistant strains of Acinetobacter baumannii. Antimicrob Agents Chemother. 2010; 54:5316-22. [PubMed: 20876375]

27. Wareham DW, Gordon NC, Hornsey M. In vitro activity of teicoplanin combined with colistin versus multidrug-resistant strains of Acinetobacter baumannii. J Antimicrob Chemother. 2011; 66:1047-51. [PubMed: 21393131]

28. Li J, Nation RL, Owen RJ, Wong S, Spelman D, Franklin C. Antibiograms of multidrug-resistant clinical Acinetobacter baumannii: promising therapeutic options for treatment of infection with colistin-resistant strains. Clin Infect Dis. 2007; 45:594-8. [PubMed: 17682994]

29. Pournaras S, Vrioni G, Neou E, et al. Activity of tigecycline alone and in combination with colistin and meropenem against Klebsiella pneumoniae carbapenemase (KPC)-producing

Enterobacteriaceae strains by time-kill assay. Int J Antimicrob Agents. 2011; 37:244-7. [PubMed: 21236643]

30. Clancy CJ, Chen L, Hong JH, et al. Mutations of the ompK36 porin gene and promoter impact responses of sequence type 258, KPC-2-producing Klebsiella pneumoniae strains to doripenem and doripenem-colistin. Antimicrob Agents Chemother. 2013; 57:5258-65. [PubMed: 23939888]

31. Souli M, Rekatsina PD, Chryssouli Z, Galani I, Giamarellou H, Kanellakopoulou K. Does the activity of the combination of imipenem and colistin in vitro exceed the problem of resistance in metallo-beta-lactamase-producing Klebsiella pneumoniae isolates? Antimicrob Agents Chemother. 2009; 53:2133-5. [PubMed: 19258266] 
32. Tangden T, Hickman RA, Forsberg P, Lagerback P, Giske CG, Cars O. Evaluation of double- and triple-antibiotic combinations for VIM- and NDM-producing Klebsiella pneumoniae by in vitro time-kill experiments. Antimicrob Agents Chemother. 2014; 58:1757-62. [PubMed: 24395223]

33. Bergen PJ, Tsuji BT, Bulitta JB, et al. Synergistic killing of multidrug-resistant Pseudomonas aeruginosa at multiple inocula by colistin combined with doripenem in an in vitro pharmacokinetic/pharmacodynamic model. Antimicrob Agents Chemother. 2011; 55:5685-95. [PubMed: 21911563]

34. Lee HJ, Bergen PJ, Bulitta JB, et al. Synergistic activity of colistin and rifampin combination against multidrug-resistant Acinetobacter baumannii in an in vitro pharmacokinetic/ pharmacodynamic model. Antimicrob Agents Chemother. 2013; 57:3738-45. [PubMed: 23716052]

35. Deris ZZ, Yu HH, Davis K, et al. The combination of colistin and doripenem is synergistic against Klebsiella pneumoniae at multiple inocula and suppresses colistin resistance in an in vitro pharmacokinetic/pharmacodynamic model. Antimicrob Agents Chemother. 2012; 56:5103-12. [PubMed: 22802247]

36. Plachouras D, Karvanen M, Friberg LE, et al. Population pharmacokinetic analysis of colistin methanesulphonate and colistin after intravenous administration in critically ill patients with gramnegative bacterial infections. Antimicrob Agents Chemother. 2009; 53:3430-6. [PubMed: 19433570]

37. Lora-Tamayo J, Murillo O, Bergen PJ, et al. Activity of colistin combined with doripenem at clinically relevant concentrations against multidrug-resistant Pseudomonas aeruginosa in an in vitro dynamic biofilm model. J Antimicrob Chemother. 2014; 69:2434-42. [PubMed: 24833752]

38. Qureshi ZA, Paterson DL, Potoski BA, et al. Treatment outcome of bacteremia due to KPCproducing Klebsiella pneumoniae: superiority of combination antimicrobial regimens. Antimicrob Agents Chemother. 2012; 56:2108-13. [PubMed: 22252816]

39. Zarkotou O, Pournaras S, Tselioti P, et al. Predictors of mortality in patients with bloodstream infections caused by KPC-producing Klebsiella pneumoniae and impact of appropriate antimicrobial treatment. Clin Microbiol Infect. 2011; 17:1798-803. [PubMed: 21595793]

40. Tumbarello M, Viale P, Viscoli C, et al. Predictors of mortality in bloodstream infections caused by Klebsiella pneumoniae carbapenemase-producing $K$. pneumoniae: importance of combination therapy. Clin Infect Dis. 2012; 55:943-50. [PubMed: 22752516]

41. Dubrovskaya Y, Chen TY, Scipione MR, et al. Risk factors for treatment failure of polymyxin B monotherapy for carbapenem-resistant Klebsiella pneumoniae infections. Antimicrob Agents Chemother. 2013; 57:5394-7. [PubMed: 23959321]

42. Linden PK, Kusne S, Coley K, Fontes P, Kramer DJ, Paterson D. Use of parenteral colistin for the treatment of serious infection due to antimicrobial-resistant Pseudomonas aeruginosa. Clin Infect Dis. 2003; 37:e154-60. [PubMed: 14614688]

43. Furtado GHC, d'Azevedo PA, Santos AF, Gales AC, Pignatari ACC, Medeiros EAS. Intravenous polymyxin $\mathrm{B}$ for the treatment of nosocomial pneumonia caused by multidrug-resistant Pseudomonas aeruginosa. Int J Antimicrob Agents. 2007; 30:315-9. [PubMed: 17631984]

44. Dalfino L, Puntillo F, Mosca A, et al. High-dose, extended-interval colistin administration in critically ill patients: is this the right dosing strategy? A preliminary study. Clin Infect Dis. 2012; 54:1720-6. [PubMed: 22423120]

45. Markou N, Markantonis SL, Dimitrakis E, et al. Colistin serum concentrations after intravenous administration in critically ill patients with serious multidrug-resistant, gram-negative bacilli infections: a prospective, open-label, uncontrolled study. Clin Ther. 2008; 30:143-51. [PubMed: 18343250]

46. Falagas ME, Rafailidis PI, Ioannidou E, et al. Colistin therapy for microbiologically documented multidrug-resistant Gram-negative bacterial infections: a retrospective cohort study of 258 patients. Int J Antimicrob Agents. 2010; 35:194-9. [PubMed: 20006471]

47. Elias LS, Konzen D, Krebs JM, Zavascki AP. The impact of polymyxin B dosage on in-hospital mortality of patients treated with this antibiotic. J Antimicrob Chemother. 2010; 65:2231-7. [PubMed: 20685752] 
48. Falagas ME, Rafailidis PI, Matthaiou DK, Virtzili S, Nikita D, Michalopoulos A. Pandrug-resistant Klebsiella pneumoniae, Pseudomonas aeruginosa and Acinetobacter baumannii infections: characteristics and outcome in a series of 28 patients. Int J Antimicrob Agents. 2008; 32:450-4. [PubMed: 18768302]

49. Falagas ME, Kasiakou SK, Kofteridis DP, Roditakis G, Samonis G. Effectiveness and nephrotoxicity of intravenous colistin for treatment of patients with infections due to polymyxinonly-susceptible (POS) gram-negative bacteria. Eur J Clin Microbiol Infect Dis. 2006; 25:596-9. [PubMed: 16955249]

50. Tsioutis C, Kritsotakis EI, Maraki S, Gikas A. Infections by pandrug-resistant gram-negative bacteria: clinical profile, therapeutic management, and outcome in a series of 21 patients. Eur $\mathbf{J}$ Clin Microbiol Infect Dis. 2010; 29:301-5. [PubMed: 20063025]

51. Pintado V, San Miguel LG, Grill F, et al. Intravenous colistin sulphomethate sodium for therapy of infections due to multidrug-resistant gram-negative bacteria. J Infect. 2008; 56:185-90. [PubMed: 18280570] 

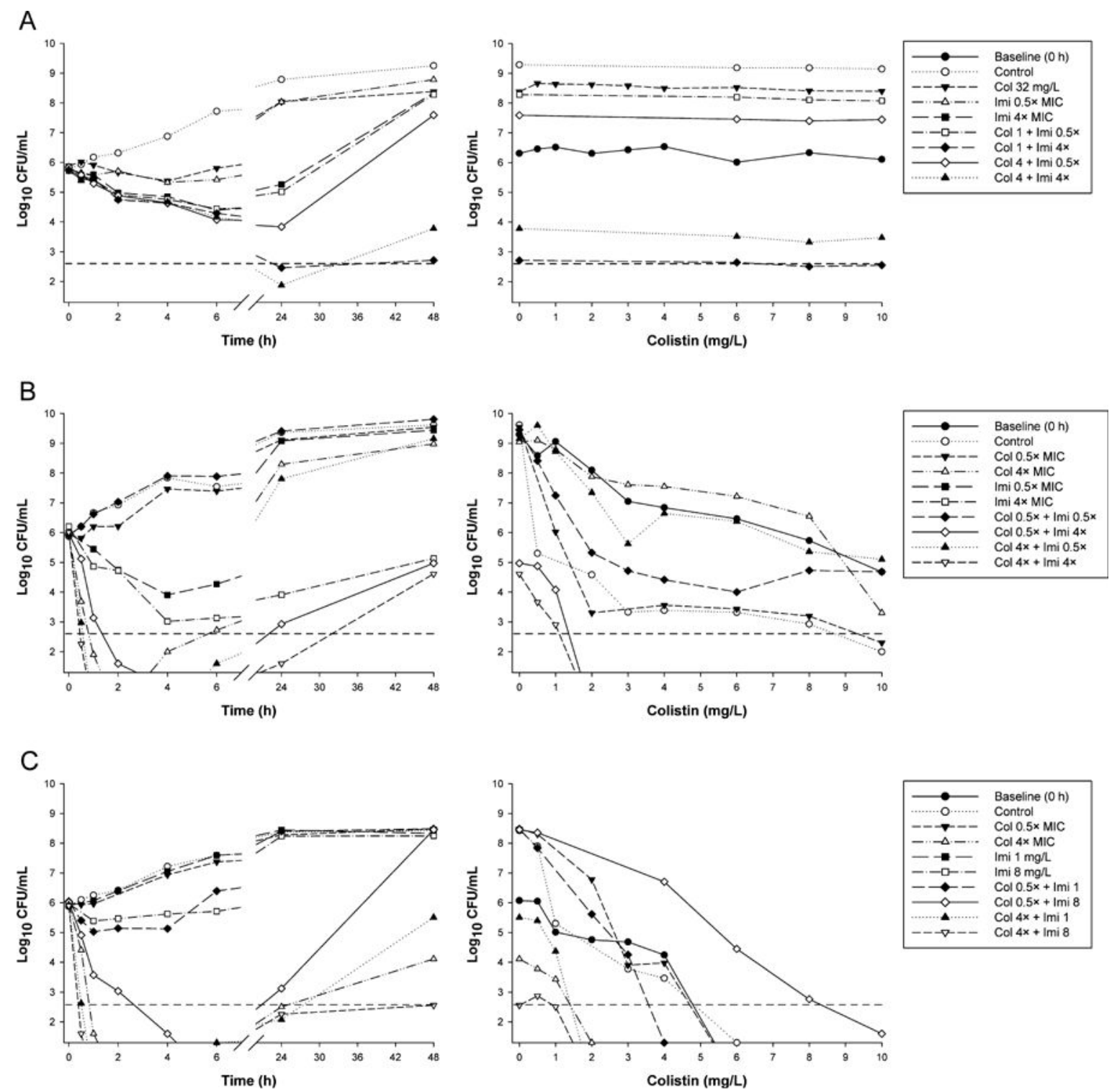

Figure 1.

Representative time-kill curves (left panels) with various clinically achievable concentrations of colistin $(\mathrm{Col})$ and imipenem alone and in combination at an inoculum of $\sim 10^{6}$ colony-forming units (CFU)/ml against three strains of Pseudomonas aeruginosa: (A) $19,147 \mathrm{n} / \mathrm{m}$ (colistin-resistant, imipenem-susceptible, multidrug-resistant (MDR), (B) 20,509 $\mathrm{n} / \mathrm{m}$ (colistin- and imipenem-susceptible, non-MDR) and (C) 20,891 n/m (colistinsusceptible, imipenem-resistant, MDR). Right panels show the respective population analysis profiles (PAPs) at baseline ( 0 hour) and after 48 hours of exposure to colistin monotherapy, colistin/imipenem combination therapy, or neither antibiotic (control). The y axis starts from the limit of detection, and the limit of quantification (LOQ) is indicated by the horizontal broken line. (Adapted from reference 20 with permission.) 


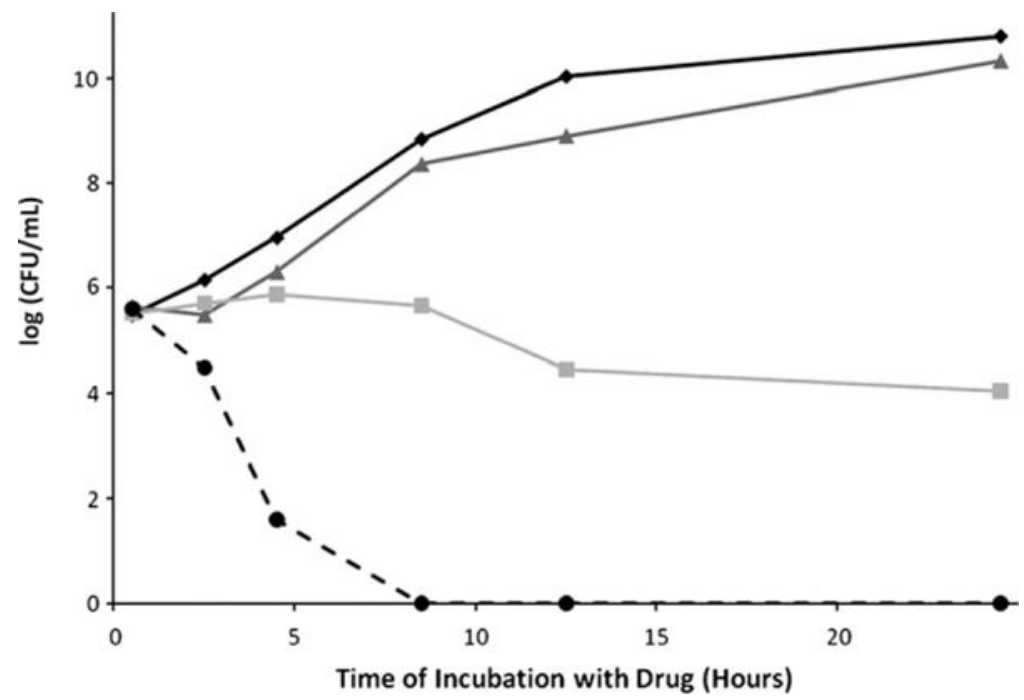

Figure 2.

Representative time-kill curves with colistin and doripenem alone, and in combination, against an extensively drug-resistant (XDR) isolate of Acinetobacter baumannii. (Adapted from reference 23 with permission.) 

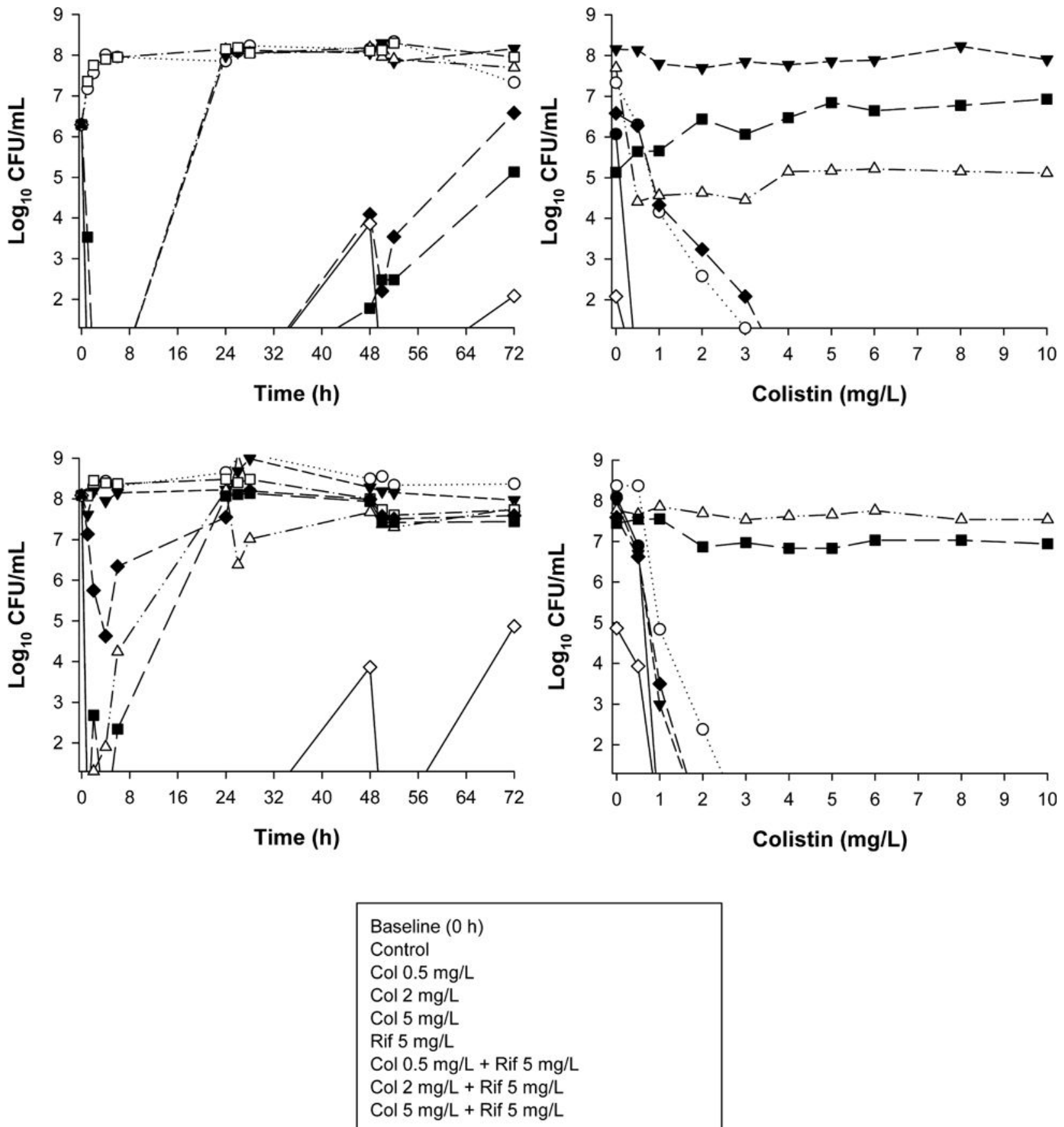

Figure 3.

Left panels: Time-kill curves with various clinically relevant dosage regimens of colistin (Col) and rifampin (Rif) alone and in combination at an inoculum of $\sim 10^{6}$ colony-forming units CFU/ml (panel A) and $\sim 10^{8} \mathrm{CFU} / \mathrm{ml}$ (panel B) against an MDR colistin-susceptible clinical isolate (FADDI-AB030) of Acinetobacter baumannii. Right panels: Population analysis profiles (PAPs) at baseline $(0 \mathrm{hr})$ and after 72 hours of exposure to colistin monotherapy, colistin-rifampin combination therapy, or neither antibiotic (control). (Adapted from reference 34 with permission.) 
A

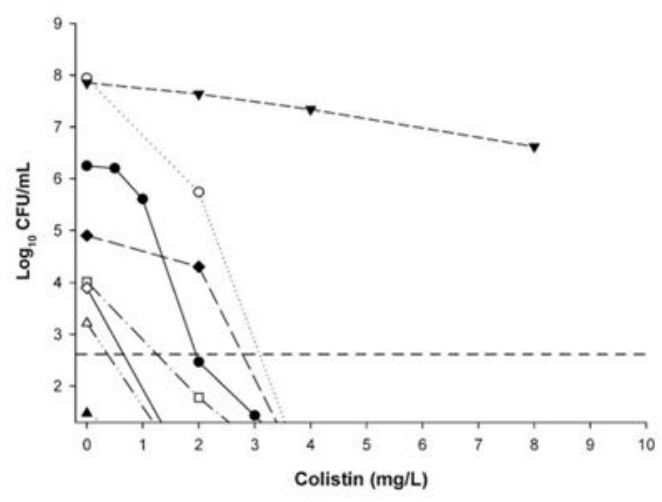

C

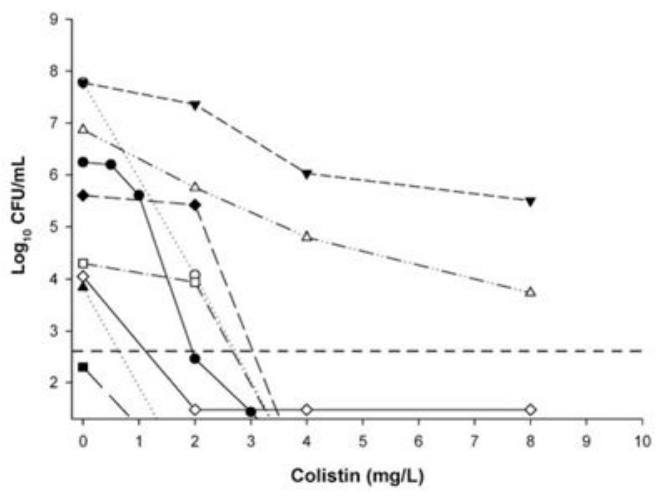

E

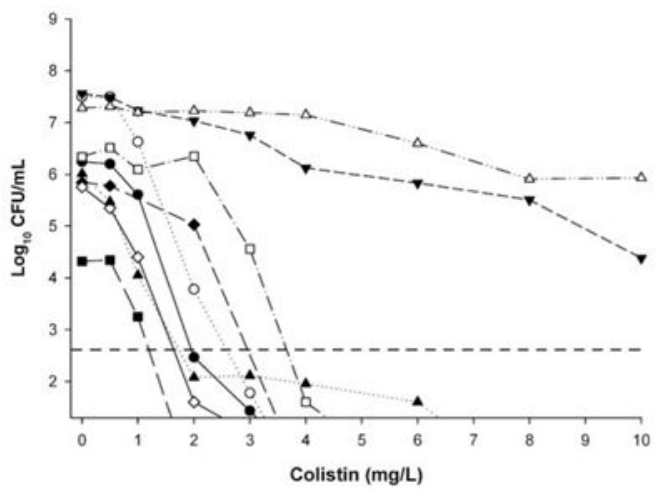

B
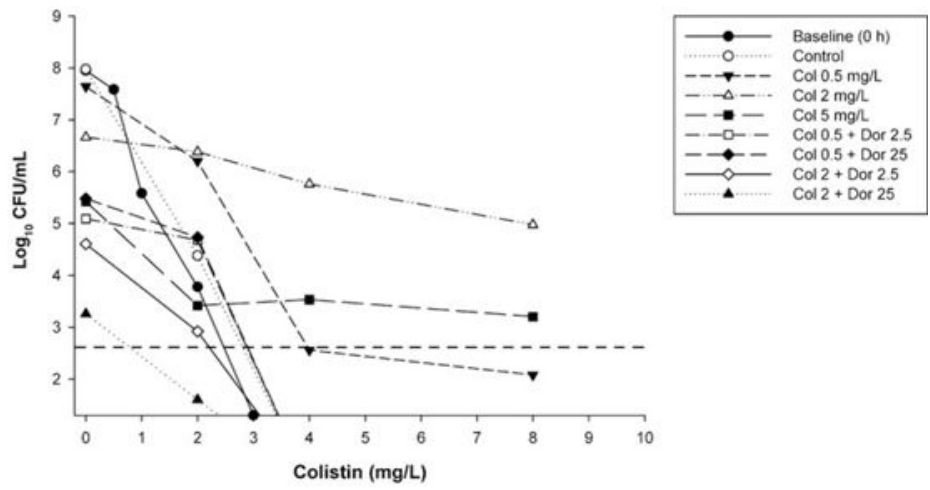

D
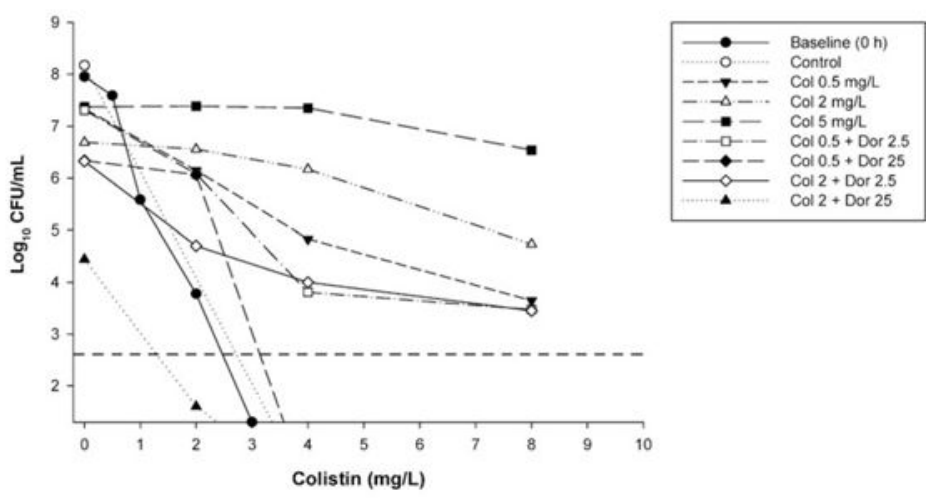

$\mathbf{F}$

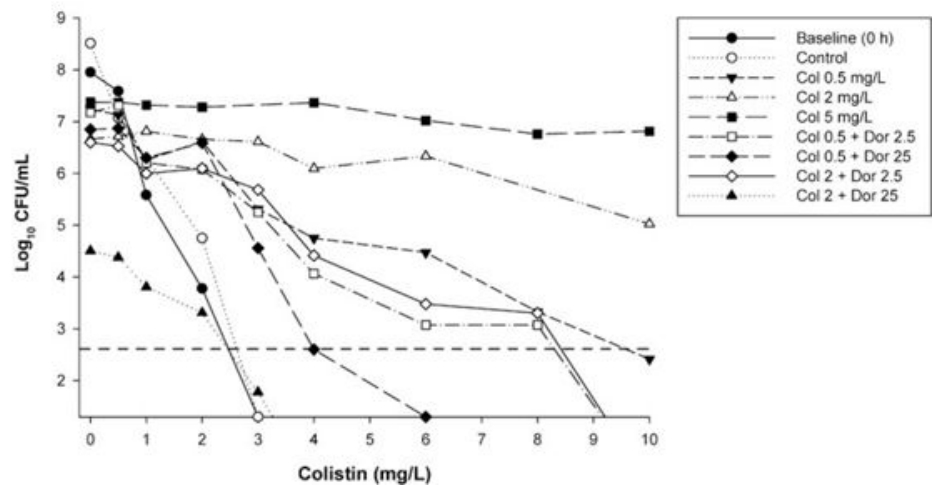

Figure 4.

Population analysis profiles (PAPs) against Pseudomonas aeruginosa ATCC 27853 with colistin monotherapy, colistin plus doripenem combination therapy, or neither antibiotic (control) at $10^{6}$ colony-forming units $\mathrm{CFU} / \mathrm{ml}$ inoculum (left panels) and $10^{8} \mathrm{CFU} / \mathrm{ml}$ inoculum (right panels), at 24 hours (panels A and B), 72 hours (panels C and D), and 96 hours (panels $\mathrm{E}$ and F). Baseline (0-hr) PAPs are shown in all panels. Colonies growing on $\geq 4 \mathrm{mg} / \mathrm{L}$ colistin are considered resistant. The y axis starts from the limit of detection, and 
the limit of quantification (LOQ) is indicated by the horizontal dashed line. (Adapted from reference 33 with permission.) 